\title{
CONTRIBUIÇÕES DA CAMINHADA COMO ATIVIDADE FÍSICA DE LAZER PARA IDOSOS
}

\author{
Recebido em: 16/02/2011 \\ Aceito em: 01/12/2011 \\ Rodrigo de Rosso Krug \\ Moane Marchesan \\ Júlio César Rodrigues da Conceição \\ Giovana Zarpellon Mazo \\ Gabriel Aguiar Antunes \\ Jamile Centenaro Romitti \\ Universidade do Estado de Santa Catarina - UESC \\ Florianópolis - Santa Catarina - Brasil
}

RESUMO: Este estudo de caso fenomenológico analisou as contribuições da caminhada como atividade física de lazer na percepção de 11 idosos. Utilizaram-se entrevistas semiestruturadas que foram interpretadas através da análise fenomenológica. Motivos que levaram os idosos a realizar a caminhada: indicação médica, benefícios para à saúde, gosto pela caminhada, orientação especializada, necessidade de praticar a caminhada regularmente, estética, e indicação profissional. Benefícios da caminhada: aumento da disposição, da alegria, manutenção da aptidão física, melhora do bem-estar, humor, da pressão arterial, da artrose e artrite, fortalecimento muscular, estética, diminuição das dores nas pernas e sociabilização. A caminhada realizada no lazer contribui tanto na capacidade funcional, quanto no estado psicológico e social desta população.

PALAVRAS CHAVE: Idoso. Caminhada. Atividades de Lazer.

\section{WALK AS CONTRIBUTIONS OF LEISURE PHYSICAL ACTIVITY FOR ELDERLY}

ABSTRACT: This phenomenological case study examined the contributions of walking as leisure physical activity in the perception of the elderly 11 . We used semistructured interviews which were interpreted by phenomenological analysis. Reasons for the elderly to do the walk: prescription and health benefits, taste for walking, expert guidance, need to practice walking regularly, aesthetics, and professional statement. Benefits of walking: increase in provision, joy, maintaining physical fitness, general well-being, mood, blood pressure, osteoarthritis and arthritis, muscle strength, aesthetics, reduction of leg pain and socialization. The walk held at leisure contributes both in functional capacity, and in the psychological and social status of this population. 


\section{KEYWORDS: Aged. Walking. Leisure Activities.}

\section{Introdução}

O número crescente de pessoas idosas em todo o mundo já está demonstrado em muitos estudos (KALACHE; VERAS; RAMOS, 1987; CARVALHO; GARCIA, 2003), bem como as alterações biopsicossociais do processo de envelhecimento, que colocam em risco a qualidade de vida destas pessoas por limitarem sua capacidade de realizar atividades rotineiras com vigor (SPIRDUSO, 1995).

Estas perdas progressivas decorrentes do envelhecimento humano contribuem também com o aumento do risco de inatividade física nesta população (PATE et al., 1995), tornando-se necessária à implementação de estratégias que visem à reabilitação da pessoa idosa.

Entre essas estratégias pode-se citar a prática regular de atividade física, que, se realizada de maneira adequada, contribui com a melhora e/ou manutenção da capacidade física (CAROMANO; IDE; KERBAUY, 2006), estando também associada ao aumento da qualidade de vida (MATSUDO; MATSUDO; BARROS NETO, 2001). Além disso, estudos mostram que pessoas idosas que praticam atividades físicas estão psicologicamente e socialmente melhores que as que não realizam esta prática (MAZO, 2008; MATSUDO; MATSUDO; BARRSO NETO, 2001).

Uma das atividades físicas de lazer mais praticadas pela população idosa é a caminhada (HUGHES et al., 2008). Essa atividade deve ser recomendada por ser um movimento natural, de fácil realização e de ótimos resultados, podendo ser realizada para promover a saúde, o condicionamento físico e o lazer (MAZO; LOPES; BENEDETTI, 2009), sendo o último muito importante para esta população. 
A prática de uma atividade física de lazer pode ocasionar a melhora da capacidade funcional ( RYDWIK; FRANDIN; AKNER, 2004), dos aspectos psicológicos (WARBURTON; NICOL; BREDIN, 2006) e sociais (WEINBERG; GOULD, 2001).

Os benefícios desta prática para pessoas idosas já são comentados na literatura (MAZO, 2008). No entanto, a literatura carece de informações sobre os benefícios da caminhada como atividade física de lazer sobre o ponto de vista das pessoas idosas praticantes desta modalidade.

Desta maneira, o presente estudo objetivou analisar as contribuições da caminhada como atividade física de lazer na percepção dos idosos que a praticam no projeto de atividades físicas do Grupo de Estudos da Terceira Idade (GETI) da Universidade do Estado de Santa Catarina (UDESC), proporcionando a literatura uma visão não do pesquisador, mas sim do sujeito investigado.

\section{Método}

Esta pesquisa foi aprovada no Comitê de Ética em Pesquisas em Seres Humanos da UDESC em 20 de dezembro de 2007 (processo $n^{\circ}$. 185/07).

Esta investigação caracterizou-se por ser de abordagem qualitativa com enfoque fenomenológico. Conforme Triviños (1987) "a pesquisa qualitativa de natureza fenomenológica surge como forte reação contrária ao enfoque positivista, privilegiando a consciência do sujeito e entendendo a realidade social como uma construção humana”.

Triviños (1987) explica que, para a realização de uma pesquisa qualitativa do tipo fenomenológico não é necessário, por exemplo, a elaboração de hipóteses empiricamente testadas, de testes estatísticos, tampouco de esquemas rígidos de 
Rodrigo de R. Krug, Moane Marchesan,

trabalho, determinados aprioristicamente. Antes, sim, é importante que o pesquisador desenvolva certa dose de flexibilidade, no sentido de re-elaborar, substituir, ou mesmo suprimir formulações, de acordo com as evidências e resultados que vai obtendo ao longo da pesquisa.

Segundo Koogan e Larousse (1990) “a fenomenologia é o estudo descritivo de um conjunto de fenômenos". A intenção da fenomenologia é de não separar o homem e o mundo, mas reuni-los na estrutura da experiência intencional.

Esta investigação também assumiu a forma de estudo de caso, pois, segundo Goode e Hatt (1968): o caso tem interesse naquilo que ele tem de único, de particular, mesmo que posteriormente fiquem evidentes certas semelhanças com outros casos ou situações.

Participaram desta investigação 11 pessoas com idade igual ou superior a 60 anos praticantes de caminhada no projeto de atividades físicas do GETI/UDESC, sendo desses, 8 mulheres e 3 homens. A amostra foi selecionada de forma intencional e só participaram do estudo os que se propuseram a responder a entrevista, e assinaram o termo de consentimento livre esclarecido.

A caminhada era realizada na pista de atletismo do Centro de Ciências da Saúde e do Esporte - CEFID/UDESC, nas segundas, quartas e sextas-feiras durante uma hora, e era ministrada por um acadêmico de Educação Física com supervisão de um profissional habilitado.

Neste estudo foi utilizado na coleta de informações, a entrevista semiestruturada, que para Triviños (1987) é aquela que "parte de certos questionamentos básicos, apoiados em teorias e hipóteses, que interessam à pesquisa, e que, em seguida, 
oferecem amplo campo de interrogativas, fruto de novas hipóteses que vão surgindo, à medida que se recebem as respostas do informante".

As questões norteadoras que compuseram a entrevista semi-estruturada foram às seguintes: Quais motivos que levaram o senhor (a) a praticar a caminhada no seu tempo de lazer? Quais benefícios da caminhada para o senhor (a)?

Para a coleta de dados primeiramente entrou-se em contato com a coordenadora do GETI/UDESC para pedir permissão para a realização da pesquisa. Logo após foram contatados o acadêmico que ministrava as aulas de caminhada e o profissional habilitado supervisor da modalidade, para relatar sobre a pesquisa. Em seguida contataram-se os idosos matriculados na caminhada do GETI/UDESC para explicar os objetivos e os princípios éticos da pesquisa. Os idosos que se propuseram participar do estudo assinaram o Termo de Consentimento Livre e Esclarecido - TCLE em duas vias, ficando uma de posse dos idosos e outra dos pesquisadores. A coleta de dados foi realizada logo após a prática da caminhada na pista de atletismo do CEFID/UDESC.

$\mathrm{Na}$ interpretação das informações foi utilizado método fenomenológico que, segundo Giorgi (1986), possui quatro procedimentos. São eles:

1) O sentido do todo - equivale à etapa da 'descrição do fenômeno'. A descrição é um modo de acesso à situação conforme vivida pelo sujeito e o pesquisador está presente na situação, através do meio oferecido pela linguagem. Essa linguagem pode ser de forma oral, através de entrevistas gravadas e depois, transcritas em forma de escrita através de documentos;

2) As unidades de significado - a partir do sentido do todo, obtido pela descrição do fenômeno, processa-se a 'redução fenomenológica'. Essa é também tomada como instrumento metodológico que fornece à pessoa a possibilidade de descrever a 
experiência sem os pressupostos metafísicos do senso comum, na atitude natural. Captase o sentido do todo e, então, volta-se ao inicio para discriminar as unidades de significado, conforme uma determinada perspectiva e com o foco no fenômeno que está sendo investigado;

3) Transformações das unidades de significado - para a fenomenologia toda realidade é tida como significativa, pois só o que tem sentido é real. O mundo é aquilo que percebemos. Ao atingirmos a essência do fenômeno ele se mostra em toda a sua significação. Retomamos todas as unidades, reescrevendo-as em função do fenômeno que está sendo investigado, ou seja, fazendo uma 'transformação das unidades de significado em linguagem educacional'; e,

4) Síntese das estruturas de significado - partindo-se da valorização da verdade como critério fenomenológico que está presente na essência do fenômeno, 'elabora-se uma descrição harmoniosa e consistente que equivale à própria estrutura da experiência vivida pela pessoa'. Transformar as unidades de significado em unidades estruturais de modo mais geral do que as do sujeito, de tal modo que respeitando sua experiência, possa transmitir o fenômeno como objeto de análise. Realiza-se, então a síntese de todas as unidades transformada em uma perspectiva consistente.

\section{Resultados e discussão}

Tendo em vista que as pessoas idosas em sua maioria não se encontram mais engajadas nas atividades de trabalho, e tem suas atividades domesticas reduzidas, a atividade física praticada no lazer se torna fundamental para esta população (ZAITUNE et al., 2007). Sendo assim, é muito importante verificar quais são os motivos que levam essas pessoas a aderirem a programas de atividades físicas, visto que, com o aumento da 
idade há uma redução no nível de atividade física (MATSUDO; MATSUDO; BARROS

NETO, 2001).

\section{A) Motivos que levaram as pessoas idosas a praticar a caminhada como atividade física de lazer no GETI/UDESC:}

\section{Indicação médica (4 ocorrências)}

A primeira essência dos motivos que levaram as pessoas idosas a praticar a caminhada foi a "indicação médica". Os depoimentos foram: "Foi indicação da minha pneumo porque tenho bronquite crônica (...)” (Idoso4); “(...) o médico falou que (...) deveria fazer exercício (...)” (Idoso7); “O médico disse que eu tinha que fazer exercício com urgência (...)” (Idoso 8); “Porque sou cardíaco e o médico indicou (...)” (Idoso 5).

Em relação a esta essência, Leite (2000) e Powers e Howley (2006) explicam que antes da pessoa idosa se inserir em um programa de atividades físicas regulares é recomendado que esse passe por um check-up médico. Posteriormente a pessoa idosa deve começar o programa de atividades físicas com intensidade leve, duração breve e frequência de 2 a 3 vezes por semana.

No estudo de Meurer (2010) todas as pessoas idosas tiveram recomendação médica do centro de saúde para iniciarem o programa de atividades físicas do projeto Floripa Ativa - Fase B, fato que pode ter influenciado nas respostas, pois a maioria (82\%) relatou que a recomendação médica foi o principal motivo de ingresso no programa. Também Cohen-Mansfield et al. (2004) analisaram 324 idosos com idades entre 74 e 85 anos, e verificaram que quase $70 \%$ dos participantes foram influenciados pelo aconselhamento médico para praticarem atividades físicas regularmente. 


\section{Benefícios da caminhada para saúde (4)}

Outra essência dos motivos que levaram as pessoas idosas a praticar a caminhada foi os "benefícios da caminhada em prol da saúde". Os participantes relataram o seguinte: “(...) faz bem pra saúde” (Idoso4); “porque tenho diabetes e pressão alta, pra melhorar isso comecei a caminhar (...)” (Idoso9); “ porque tenho artrite reumatóide (...) para não atrofiar" (Idoso10); "porque enferruja se não fizer nada (..), piora a saúde” (Idoso3).

Para justificar esta essência nos reportamos a Weinberg e Gould (2001), onde estes afirmam que as pessoas sentem-se motivadas para praticar atividades físicas porque sabem os benefícios fisiológicos e psicológicos que essa pode proporcionar para a saúde.

Matsudo et al. (2001) explica que a prática regular de atividades físicas proporciona benefícios à saúde sendo estes referentes tanto aos aspectos antropométricos, neuromusculares, metabólicos como psicológicos. Os efeitos metabólicos são os aumentos do volume sistólico; da potência aeróbia; da ventilação pulmonar; a melhora do perfil lipídico; a diminuição da pressão arterial; a melhora da sensibilidade à insulina e a diminuição da freqüência cardíaca em repouso e no trabalho submáximo. Com relação aos efeitos antropométricos e neuromusculares ocorre à diminuição da gordura corporal, o incremento da força e da massa muscular, da densidade óssea e da flexibilidade.

Warburton, Nicol e Bredin (2006) citam que uma hora de caminhada por semana já causa benefícios para saúde. Christian e Barnard (2005) acrescentam que pessoas que 
caminham por pelo menos 4 horas semanais tem uma redução em $25 \%$ no risco de ter câncer de pulmão.

\section{O gosto pela caminhada $(3)$}

Nas falas de algumas participantes deste estudo ficou evidenciada que o "gosto pela caminhada" é um dos motivos pelo qual esses praticam a referida atividade física. As falas foram: "porque eu caminhava na esteira e gostava (...)” (Idoso1); “(...) porque gosto de caminhada.” (Idoso5); “(...) já gostava de caminhar (...)” (Idoso9).

Estas informações estão em concordância com a afirmação de Miranda e Batista (1999) de que o prazer é fundamental para a aderência à atividade física, superando a necessidade do sentimento de utilidade. No estudo de Caspersen, Kriska e Dearwater (1994) a caminhada foi considerada atividade física mais praticada por pessoas acima de 60 anos.

Em estudo realizado com pessoas idosas, na cidade de Belo Horizonte, o prazer pela prática foi apontado como um motivo para esses ingressarem em um programa de atividades físicas (LINS; CORBUCCI, 2007). Os autores destacam que ter o prazer como o motivo mais referenciado para ingressar em programa de atividades físicas, é uma motivação intrínseca que evidencia o desejo pessoal associado à realização e satisfação em estar em um grupo social, com manifestações de respeito, cumplicidade e reconhecimento social, desejos que se tornam importantes, principalmente, nesta fase da vida.

Gutieres (2001) explica que o lazer esta associado à busca pessoas do prazer, sendo assim os idosos praticam a caminhada como atividade física de lazer porque sentem prazer quando a realizam. 
Rodrigo de R. Krug, Moane Marchesan,

Meurer (2010) relata que o prazer pela prática de exercício físico é um dos fatores citados pelas pessoas idosas que participaram de seu estudo como fator motivante para a aderência a prática de atividades físicas regulares.

\section{Ter orientação especializada (3)}

Uma outra essência dos motivos que levaram as pessoas idosas deste estudo a praticar a caminhada foi a "orientação especializada". Os participantes relataram o seguinte: “(...) gosto de ser orientada por alguém.” (Idoso6); “(...) preferi aqui por haver orientação e acompanhamento de professores (...)” (Idoso7); “(...) devido a orientação.” (Idoso9).

De acordo com Carvalho et al. (1996) todo o início ou reinício de atividade física deve ser controlado, principalmente para pessoas idosas. Isto evidência a importância de uma orientação especializada para a prática de atividades físicas regulares, pois segundo Ferreira (2001) a prática regular de atividade física só proporcionará benefícios para saúde se esta for bem orientada e bem praticada, devendo ser encarada como um meio potencial para se contribuir para a saúde.

Com isso evidencia-se assim a importância de se ter uma orientação especializada durante a pratica de atividades físicas, pois segundo Nahas (2006) para que os benefícios da atividade física sejam contemplados, é necessário que a prática seja fundamentada em alguns princípios básicos, como a intensidade, a duração e a frequência, conceitos estes estudados por profissionais de Educação Física. 


\section{A necessidade de praticar atividade física regularmente (2)}

Facilmente podemos desvelar nas falas de algumas pessoas idosas que o motivo pelo qual eles praticam caminhada no GETI/UDESC é a "necessidade de praticar atividade física regularmente”. Os depoimentos foram: “(...) preciso fazer alguma atividade física.” (Idoso2); “(...) a necessidade de fazer atividade física.” (Idoso3).

Esta essência é justificada pela Organização Pan-americana de Saúde (2003) que ressalta que para pessoas idosas é necessária a prática regular de atividades físicas que fortaleçam a musculatura e mantenham o equilíbrio, pois elas podem reduzir os riscos de quedas e melhor o estado funcional destas pessoas. Nahas (2006) acrescenta que a atividade física regular melhora o controle do stress, o aumento da capacidade funcional e autonomia, o aumento da satisfação e da manutenção da massa muscular, previne a osteoporose, reduz o risco de morte prematura e de desenvolvimento de doenças crônicas, auxilia o peso corporal e aumenta a auto-estima, além disso, proporciona maior capacidade de realização das atividades da vida diária, preservando assim a independência destas pessoas que estão na terceira idade.

Warburton, Nicol e Bredin (2006) relatam que hoje em dia várias organizações de saúde já defendem que um gasto energético de 1000 Kcal por semana já proporciona vários benefícios adicionais a saúde. Mais recentemente pesquisadores tem postulado que níveis ainda menores de atividade física semanal já proporcionam benefícios para a saúde, ou seja, 500 Kcal por semana seria um gasto energético semanal bom para pessoas idosas ou descondicionadas terem benefícios para a saúde. 


\section{Estética (1)}

Outra essência dos motivos pelo qual as pessoas idosas praticam caminhada é a “estética” que ficou evidenciada na fala “(...) pra não engordar.” (Idoso1).

Zanata e Fonseca (2008) explicam que a estética é muito valorizada pela sociedade, pois os perfis de artistas famosos, os hábitos e técnicas para a promoção da boa aparência são os principais assuntos abordados em redes de televisão, revistas e jornais eletrônicos, influenciando assim as pessoas a realizarem atividades físicas.

Balbinotti e Barbosa (2006) citam que querer ter ou ficar com o corpo bonito ou definido é um dos motivos pelo qual as pessoas idosas fazem atividades físicas. Duarte, Santos e Gonçalves (2002) ao avaliarem os motivos de adesão a caminhada em um grupo de pessoas idosas, verificaram que a estética é um fator motivador para tal decisão.

\section{Sociabilização (1)}

Podemos desvelar na fala “(...) vim pra fazer em grupo (...)” (Idoso1) que a "sociabilização" é uma das essências que levaram as pessoas idosas a praticar a caminhada regularmente no GETI/UDESC.

Para justificar essa essência usamos a fala de Balbinotti e Barbosa (2006) que dizem que a atividade física é vista como uma fonte de satisfação, sensação de bemestar e sociabilidade, sendo considerada uma boa oportunidade para encontrar, estar ou reunir-se com amigos. Ainda nesse sentido, Borges e Leal (2006) colocam os benefícios psicológicos e sociais como a principal motivação para caminhar. 
A sociabilização e o lazer estão intimamente relacionadas, pois de acordo com Nóbrega et al., (1999) estas são características importantes para que um programa de atividades físicas para pessoas idosas seja bem sucedido.

Meurer (2010) encontrou em seu estudo que a sociabilização, com $8,6 \%$, foi o segundo maior motivo citado pelas pessoas idosas, para a aderência ao projeto de atividades físicas Floripa Ativa - Fase B. No estudo de Cerri e Simões (2007) a sociabilidade também foi um fator importante para a os idosos aderirem à prática de atividades físicas.

\section{Indicação de um profissional de Educação Física (1)}

A ultima essência dos motivos pelos quais as pessoas idosas fazem caminhada no GETI/UDESC é a “indicação de um profissional de Educação Física”. Este motivo foi desvelado a partir da fala do Idoso11 que disse: "Um profissional de Educação

\section{Física me indicou."}

Neste sentido cabe ao profissional de Educação Física, que além de ser um educador, também é um profissional de saúde, informar as pessoas sobre os benefícios de um estilo de vida ativo sobre a mesma (NAHAS, 2006). Ressaltando a importância de praticar atividades físicas regularmente. Sendo assim o profissional de Educação Física desponta como mediador da prática de atividades físicas tendo importante papel em relação à promoção a saúde, educação e prevenção de doenças, contribuindo assim para hábitos de vida mais saudáveis (KRUG et al., 2009).

De acordo com Devide (1996) é de suma importância que o professor (profissional de Educação Física) incentive os alunos a praticar atividades físicas 
regularmente, pois isso terá grande influência sobre as ações dos alunos, pois este professor influencia seus alunos seja como pessoa seja como profissional.

Shephard (1990) afirma que o acompanhamento através de instrução de profissionais especializados apóiam o envolvimento de pessoas idosas em programas de atividades físicas.

\section{B) Benefícios da caminhada para os idosos praticantes de caminhada no GETI/UDESC:}

\section{Aumento da disposição (4)}

A primeira essência dos benefícios da caminhada para os idosos que a praticam foi o "aumento da disposição". As falas das pessoas idosas que caminham no GETI/UDESC foram as seguintes: “(...) fico mais disposto (...)” (Idoso1); “Aumenta a disposição física e mental (...)". (Idoso2); "Melhorou a disposição para fazer os trabalhos de casa (...)” (Idoso8); “(...) tenho mais disposição (...)”. (Idoso9).

Nesta essência utilizamos Duarte, Santos e Gonçalves (2002) que ao estudarem os motivos de adesão a caminhada em um grupo de pessoas idosas, concluíram que a atividade física regular proporciona maior disposição nesta população, na opinião dos próprios praticantes de caminhada.

Fato este também corroborado por Oliveira (2002) onde este diz que a prática regular de atividade física por pessoas idosas é de fundamental importância para o aumento da disposição física e mental. 
Rodrigo de R. Krug, Moane Marchesan,

Julio Cesar R. da Conceição, Giovana Z. Mazo,

Gabriel A. Antunes e Jamile C. Romitti

\section{Fortalecimento muscular (2)}

Outra essência dos benefícios da caminhada para as pessoas idosas ativas foi o "fortalecimento muscular". Os depoimentos foram os seguintes: “Bom (...) pra não atrofiar os músculos.” (Idoso1); “Fortalece a musculatura”. (Idoso4).

Esta essência é fundamentada considerando as informações expostas por Booth, Weeden e Tseng (1994), que dizem que a perda de fibras musculares, motoneurônios, unidades motoras, massa muscular e força muscular começa entre os 50 e 60 anos. De acordo com Matsudo (2002) A perda da massa muscular e conseqüentemente da força muscular é a principal responsável pela deterioração na mobilidade e na capacidade funcional do indivíduo que está envelhecendo.

Por isso da importância das pessoas idosas fazerem atividade física regularmente, pois Booth, Weeden e Tseng (1994) explicam que se estas pessoas que estão na terceira idade se manterem fisicamente ativas, essas terão somente perdas moderadas da massa muscular. Alves et al. (2004) em seu estudo com pessoas idosas que praticavam hidroginástica regularmente também evidenciaram uma aumento da força muscular em seus praticantes, tanto em membros inferiores como em membros superiores. Caetano e Tavares (2008) concordam com essa afirmação ao relatarem que a atividade física regular aumenta a força muscular.

\section{Melhora do bem-estar (2)}

A "melhora do bem-estar" foi outro beneficio da caminhada na percepção das pessoas idosas. As falas que nos levaram a chegar nessa essência foram: "Me sinto bem, a vontade, tranquilo”. (Idoso5); “(...) o bem-estar sentido (...).” (Idoso7). 
Rodrigo de R. Krug, Moane Marchesan,

Julio Cesar R. da Conceição, Giovana Z. Mazo,

Gabriel A. Antunes e Jamile C. Romitti

Nahas (2006) e Mazo (1998) relatam que as pessoas que tem um estilo de vida mais ativo, ou seja, que fazem atividades físicas regularmente apresentam maior sensação de bem-estar psicológico.

Warburton, Nicol e Bredin (2006) citam o mesmo fato ao explicarem que além de muitos outros benefícios, a prática regular de atividade física também está associada à melhora do bem estar psicológico, através da redução do stress, ansiedade e depressão.

As atividades físicas de lazer têm essa capacidade de proporcionar sensação de bem-estar e também são capazes de provocar mudanças em outros aspectos da vida das pessoas (QUEIROZ; SOUZA, 2009).

Para Cooper (1982) a atividade física aeróbica, realizado com intensidade moderada e longa duração (a partir de 30 minutos) proporciona um alívio do estresse ou tensão, devido a taxa de um conjunto de hormônios denominados endorfinas que agem sobre o sistema nervoso, reduzindo o impacto estressor do ambiente e com isso pode prevenir ou reduzir transtornos depressivos. Com isso a atividade física regular proporcionará através da diminuição do estresse e da depressão a melhora do bem-estar psicológico.

\section{Melhora do humor (1)}

A "melhora do humor" foi outro beneficio da caminhada que foi citado pelo Idoso7 na seguinte fala: “Melhora (...), o humor”.

Oliveira (2002) explica que em decorrência dos efeitos cumulativos do envelhecimento sobre a função do sistema nervoso central, algumas alterações no metabolismo protéico são observadas, acarretando uma perda neuronal, uma redução das concentrações dos neurotransmissores e consequentemente a fisiologia do sistema 
serotoninérgico sofre algumas alterações, podendo ocasionar alterações de humor nas pessoas mais idosas.

De acordo com Borges, Benedetti e Mazo (2010), Caetano e Tavares (2008) e Mazo, Lopes e Benedetti (2009) a atividade física regular proporciona a seus praticantes uma menor incidência de humor deprimido, ou seja, melhora o humor das pessoas mais ativas fisicamente.

Iwasaki e Mannell (2000) enfatizam que as atividades físicas de lazer melhoram o humor das pessoas, pois estas tem o potencial de reduzir o stress diminuindo o humor negativo e aumentando o humor positivo de quem realiza esta pratica regularmente.

\section{Aumento da alegria (1)}

Outra essência das contribuições da caminhada na opinião das pessoas idosas que a praticam no GETI/UDESC é o aumento da alegria que ficou evidenciada na seguinte colocação: “Aumenta a (...), alegria”. (Idoso3).

Este beneficio da caminhada citado por um de seus praticantes é justificado pela fala de Guedes (1996) quando este relata que a prática de atividade física proporciona uma maior alegria de viver em seus praticantes. De acordo com Loret (1994) as pessoas realizam atividades físicas de lazer em busca de estimulação, excitação e principalmente de alegria.

Allsen, Harrison e Vance (2001) explicam que a prática regular de atividades físicas dão oportunidade de seu praticante ter uma sensação de sucesso, aumentando sua autoestima e alegria. Lima (2001) também relata este fato ao explicar que as pessoas idosas que praticam esportes, ginástica e dança, demonstram uma maior vitalidade e alegria. 


\section{Melhora da artrose e da artrite (1)}

A partir da fala do Idoso6 ficou evidenciada que a "melhora da artrose e da artrite" foi outra contribuição da caminhada para seus praticantes idosos. A fala foi: “É excelente pra artrose e artrite (...)”.

Das perdas físicas, as mioarticulares são as mais fáceis de serem percebidas durante o envelhecimento, a exemplo do enfraquecimento muscular, cuja causa é multifatorial (CAROMANO et al., 2007). De acordo com Mazo, Lopes e Benedetti (2009) a artrose e a artrite são patologias mais freqüentes em pessoas com idade superior a 40 anos, sendo que a atividade física é sugerida como tratamento, para não ocorrer atrofia das articulações atingidas por falta de mobilidade e para atenuação da dor. As mesmas autoras explicam que ultimamente a caminhada é muito recomendada para pessoas com artrose e/ou artrite.

Mesmo iniciado em uma fase tardia da vida, a prática de atividades físicas para pessoas idosas ocasionam benefícios à saúde, inclusive para indivíduos portadores de doenças crônicas, prevenindo e/ou ajudando a tratar principalmente as doenças associadas à inatividade física, como é o caso da artrose e da artrite (ELIOT; LONG; BOONE, 1992). A atividade física regular segundo Stella et al. (2002) melhora o desempenho das articulações, devido a melhora da massa muscular e da massa óssea (STELLA et al., 2002), podendo prevenir ou tratar a artrite (MARQUES; CONDO, 1998). 
Rodrigo de R. Krug, Moane Marchesan,

Julio Cesar R. da Conceição, Giovana Z. Mazo,

Gabriel A. Antunes e Jamile C. Romitti

\section{Manutenção da aptidão física (1)}

Outra essência muito relata pelas pessoas idosas como beneficio da caminhada é a "manutenção da aptidão física". Os depoimentos que fizeram emergir esta essência foram: “A caminhada é boa para não encarangar, não enferrujar, pra melhorar a condição física." (Idoso11).

A queda da aptidão física com o envelhecimento é fato comprovado, que se inicia de maneira gradativa, ao redor da quinta década de vida. Entretanto Alves et al. (2004) diz que vários estudos apontam para os benefícios dos programas de atividades físicas para pessoas idosas, como medida importante para retardar ao máximo os efeitos do envelhecimento sobre a aptidão física, contribuindo de maneira significativa para a sua manutenção.

Mcauley et al. (2000) ao estudar 174 pessoas com mais de 60 anos participantes de um programa de caminhadas ou resistência muscular evidenciou que a prática de atividades físicas colabora para melhoria da aptidão física, diminuição da gordura corporal e aumento da auto-eficácia.

De acordo com Matsudo (2002) os sujeitos mais ativos fisicamente têm menor peso corporal, índice de massa corporal, percentual de gordura e relação cintura/quadril se comparados aos indivíduos da mesma idade que são inativos fisicamente, sendo assim, esses tem uma melhor aptidão física.

\section{Melhora da estética (1)}

A décima essência relatada pelos participantes do estudo em relação aos benefícios da caminhada é a "melhora da estética". O Idoso1 relatou o seguinte: “ $A$ caminhada é boa (...), pra emagrecer”. 
A melhora da estética como benefício da pratica regular de atividades físicas é relatada por Freitas et al. (2007) quando estes evidenciaram em seu estudo com 300 pessoas idosas que faziam atividades físicas no projeto Máster Vida da Universidade Federal de Pernambuco que a estética é um fato muito citado pelos participantes como uma contribuição da atividade física. No mesmo estudo também se evidenciou que o emagrecimento com 48,3\%, é considerado uma importantíssima contribuição da atividade física na opinião das pessoas idosas que participaram da pesquisa. (48,3\%).

Duarte, Santos e Gonçalves (2002) também acharam resultados que vão ao encontro deste estudo, ao estudarem o motivo de adesão de pessoas idosas a pratica de caminhada na cidade de Pelotas-RS, pois essas salientaram que a estética é um grande benefício da prática de caminhada.

\section{Diminuição das dores nos membros inferiores (1)}

Outra contribuição da caminhada na opinião destas pessoas que a realizam é a “diminuição das dores nos membros inferiores”. O depoimento foi o seguinte: “(...) a melhora das dores nas pernas, (...)”. (Idoso7).

No estudo experimental de Silva e Lima (2002) com 33 sujeitos diabéticos inativos fisicamente, com idades entre 45 e 72 anos, onde esses foram submetidos a um programa de atividades físicas, com 10 semanas de duração, sendo 4 sessões por semana de 60 minutos cada, sendo 5 minutos de aquecimento (alongamento), 40 minutos de exercícios aeróbios (caminhada, corrida ou bicicleta), 10 minutos de exercícios de resistência muscular localizada (com pesos) e 5 minutos de resfriamento (alongamento e relaxamento), conclui-se que a atividade física regular diminui as dores no corpo. 
Rodrigo de R. Krug, Moane Marchesan,

Contribuições da Caminhada como Atividade Física...

Julio Cesar R. da Conceição, Giovana Z. Mazo,

Gabriel A. Antunes e Jamile C. Romitti

Matsudo e Matsudo (1992) também cita em sua pesquisa de revisão de literatura que a atividade física se mostra benéfica na diminuição de dores crônicas para quem a pratica regularmente.

\section{Sociabilização (1)}

A "sociabilização" também foi citada pelos participantes deste estudo como um beneficio da caminhada. A fala foi a seguinte: “(...) a convivência melhora muito, a amizade.” (Idoso9).

De acordo com Weinberg e Gould (2001) a perda do espaço social é mais um problema do envelhecimento humano, principalmente, após o evento da aposentadoria. Estes autores acrescentam que os programas de atividades físicas podem amenizar essa perda, pois representam uma maior convivência entre amigos. Meurer (2010) também cita isso, quando diz que o fator sociabilização dentro de um grupo de atividades físicas é muito importante, pois pode fazer com que os participantes tenham uma perspectiva de novas relações de amizades.

Cardoso (1992) segue esta linha de pensamento ao relatar que a atividade física além de poder atenuar e retardar o processo de declínio das funções orgânicas que são decorrentes do envelhecimento humano pode também promover melhoras nas habilidades sociais das pessoas e levar estas a uma maior participação social.

A sociabilização é muito visada e ampliada pelos programas de atividades físicas de lazer voltados para as pessoas mais velhas. As atividades físicas de lazer propiciam novas vivencias e representam para as pessoas idosas uma forma de sociabilização (FENALTI; SCHWARTZ, 2003). 
Rodrigo de R. Krug, Moane Marchesan,

Julio Cesar R. da Conceição, Giovana Z. Mazo,

Contribuições da Caminhada como Atividade Física...

Gabriel A. Antunes e Jamile C. Romitti

\section{Melhora da pressão arterial (1)}

Uma importante contribuição da caminhada para as pessoas idosas na opinião das mesmas foi a "melhora da pressão arterial". Esta essência ficou evidenciada na fala do Idoso9 que foi: “(...) normaliza a pressão (...)”.

Christian e Barnard (2005) citam que a atividade física regular diminui o risco da doença arterial incluindo os efeitos nos lipídios plasmáticos, na função endotelial, a diminuição da sensibilidade à insulina e a diminuição da pressão arterial. De acordo com Haskell et al. (2007) e com a Organização Mundial da Saúde (2002), a atividade física se praticada regularmente pode diminuir o risco e ou a evolução de muitas doenças, dentre elas hipertensão.

Vatten, Nilsen e Holmen (2006) analisaram 30.597 mulheres e 30.508 homens, e verificaram que pessoas que fazem atividade física têm uma pressão arterial mais controlada se comparadas a pessoas inativas fisicamente.

As atividades físicas regulares, principalmente os exercícios aeróbios, no caso deste estudo a caminhada, são citados por Nahas (2006) como um fator marcante na prevenção e tratamento da hipertensão arterial.

\section{Nenhuma contribuição (1)}

A última essência das contribuições da caminhada na opinião das pessoas idosas que a praticam no GETI/UDESC foi “nenhuma". Esta ficou evidenciada pela fala do Idoso2 que disse: "Não notei nenhuma contribuição porque arrecem comecei a fazer a caminhada". 
Rodrigo de R. Krug, Moane Marchesan,

Esta essência se justifica pela própria fala da pessoa idosa que relata que arrecem começou a praticar a caminhada no GETI/UDESC, por isso ainda não obteve benefícios.

\section{Conclusão}

Após o desvelamento de todos os depoimentos dos participaram do estudo verificou-se que a caminhada praticada no tempo de lazer contribui tanto na saúde psicológica, quanto na física de pessoas idosa. Este achado ficou evidenciado nas 11 respostas que emergiram das essências da entrevista, mostrando os benefícios que a prática da caminhada proporciona para esta população. Apenas em um relato não foi evidenciado contribuição desta prática, fato que pode ser explicado pela participante ser iniciante no programa de caminhada no GETI/UDESC.

Os benefícios da caminhada na opinião das pessoas idosas praticantes são fisiológicos (fortalecimento muscular, melhora da pressão arterial, diminuição das dores nos membros inferiores, melhora da artrose e artrite, e melhora da aptidão física e estética), psicológicos (aumento da disposição, melhora do bem-estar, melhora do humor e alegria) e sociais (aumento da sociabilização).

Sendo assim, incentivar as pessoas idosas a realizarem caminhadas como atividade física de lazer é uma estratégia eficiente para contribuir com a saúde das mesmas.

Após a análise dos achados conclui-se que o GETI/UDESC tem um papel importante para a população idosa da comunidade de Florianópolis, pois além de proporcionar programas de atividade física para os participantes, ele também contribui com as relações sociais, o que é muito importante para a terceira idade. 


\section{REFERÊNCIAS}

ALLSEN, P. E.; HARRISON, J. H.; VANCE, B. Exercício e qualidade de vida: uma abordagem personalizada. São Paulo: Manole, 2001.

ALVES, R. V. et al. Aptidão física relacionada a saúde de idosos: influencia da hidroginástica. Revista Brasileira de Medicina do Esporte, Niterói v. 10, n. 1. jan./fev, 2004.

BALBINOTTI, M. A. A; BARBOSA, M. L. L. "Inventário de Motivação à Prática Regular de Atividades Físicas (IMPRAF - 126)". Manual Técnico de Aplicação. Laboratório de Psicologia do Esporte - Universidade Federal do Rio Grande do Sul: Porto Alegre, 2006.

BORGES, L. J.; BENEDETTI, T. R. B.; MAZO, G. Z.. Influencia del ejercicio físico em los síntomas depresivos y em la aptitud funcional de ancianos em el sur de Brasil. Revista Espanhola de Geriatria e Gerontolologia, v. 45 n. 2, p. 72 -78, 2010.

BORGES, L. V.; LEAL, C. R. A. A. Caminhada e representação social. In: Simpósio de Educação do Sudeste Goiano, 5, 2006. Anais... Universidade Federal de Goiás, 2006.

BOOTH, F. W.; WEEDEN, S. H.; TSENG, B. S. Effect of aging on human skeletal muscle and motor function. Med. Sci. Sports Exerc., v. 26, p.556- 560, 1994.

CAETANO, C. M.; TAVARES, D. M.S.. Unidade de Atenção ao Idoso: atividades, mudanças no cotidiano e sugestões. Revista Eletrônica de Enfermagem, v. 10, n. 3, p. 622- 631, 2008.

CARDOSO, J. R. Atividades físicas para a terceira idade. A terceira idade, v. 5, n. 4, p. 9- 21. 1992.

CAROMANO, F. A.; IDE, M. R.; KERBAUY, R. R. Manutenção na prática de exercícios por idosos. Revista do Departamento de Psicologia, v. 18, n. 2, p. 177-192, Jul./Dez. 2006.

CAROMANO, F. A.; et al. Efeitos da caminhada no sistema músculo-esquelético estudo da flexibilidade. Revista de Terapia Ocupacional da Universidade de São Paulo, v. 18, n. 2, p. 95-101, maio/ago., 2007.

CARVALHO, J. A. M.; GARCIA, R. A. O envelhecimento da população brasileira: um enfoque demográfico. Caderno de Saúde Pública. v.19, p.725-33, 2003.

CARVALHO, T.; et al.. Posição oficial da Sociedade Brasileira de Medicina do Esporte: atividade física e saúde. Revista Brasileira de Medicina do Esporte. v. 2, n. 4, Out/Dez, 1996. 
CASPERSEN, C. J.; KRISKA, A. M.; DEARWATER, S. R. Physical activity epidemiology as applied to elderly populations. Bailliers Clin. Rheumatol. v. 8, p. 727, 1994.

CERRI, A.S; SIMÕES, R. "Hidroginástica e Idosos: por que eles praticam?". Movimento.v.13, n. 01, p.81-92, 2007.

CHRISTIAN R. K.; BARNARD, R. J. Effects of exercise and diet on chronic disease. Journal of Applied Physiology, v. 98, p. 3- 13, 2005.

COHEN-MANSFIELD, J. et al.. Socio-environmental exercise preferences among older adults. Preventive Medicine, New York, v. 38, p. 804-811, 2004.

COOPER, K. H. O programa aeróbico para o bem-estar total: exercícios - dietas equilíbrio emocional . 4. ed. Rio de Janeiro: Nórdica, 1982.

DEVIDE, F. P. Educação Física e Saúde: em busca de uma reorientação para sua práxis. Revista Movimento, Porto Alegre, v. 3, n. 5, p. 44-55, 1996.

DUARTE, C. P.; SANTOS, C. L.; GONÇALVES, A. K. A concepção de pessoas de meia-idade sobre saúde, envelhecimento e atividade física como motivação para comportamentos ativos. Revista Brasileira de Ciências do Esporte, Campinas, v. 23, n. 3, p. 35-48, 2002.

ELIOT, R. S.; LONG, D. R.; BOONE, J. L. Rehabilitation. In: . ELIOT, R.S. (Ed.). Stress and the heart. New York: Futura, 1992.

FENALTI, R. C. S.; SCHWARTZ, G. M. Universidade aberta a Terceira idade e a perspectiva de ressignificação do lazer. Revista Paulista de Educação Física. v. 17, n. 2, p. 131-141, 2003.

FERREIRA, M. S. Aptidão física e saúde na Educação Física Escolar: Revista Brasileira de Ciências do Esporte, v. 22, n. 2, p. 41- 54, jan., 2001.

FREITAS, C. M. S. M. et al. Aspectos motivacionais que influenciam a adesão e manutenção de idosos a programas de exercícios físicos. Revista Brasileira de Cineantropometria e Desempenho Humano. v. 9, n. 1, p. 92- 100, 2007.

GIORGI, A. Phenomenology and psychological research. Pittburg: Duquesne University Press, 1986.

GOODE, L; HATT, K. Métodos em pesquisa social. São Paulo: Cia. Editora Nacional, 1968.

GUEDES, D. P. Atividade física, aptidão física e saúde. In: CARVALHO, T.; GUEDES, D. P.; SILVA, J. G. (Org.). Orientações Básicas sobre Atividade Física e Saúde para Profissionais das Áreas de Educação e Saúde. Brasília: Ministério da Saúde e Ministério da Educação e do Desporto, 1996. 
GUTIERES, G. L.; Lazer e Prazer: questões metodológicas e alternativas políticas. Campinas: Ed. Autores Associados, 2001.

HASKELL, W. L. et al. Physical activity and public health readapted recommendation for adults from the American College of sports Medicine and the American Hearth Association. Circulation, n. 116, p. 1081 - 93, 2007

HUGHES, J. P. et al. Leisure-time physical activity among US adults 60 or more years of age: results from NHANES 1999-2004. Journal physical activity and health. v.5, n.3, p.347-358, 2008.

IWASAKI, Y.; MANNELL, R. Hierarchical dimensions of leisure stress coping. Leisure Sciences. v. 22, p.163-181, 2000.

KALACHE, A.; VERAS, R. P.; RAMOS, L. R. O envelhecimento da população mundial. Um desafio novo. Revista de Saúde Pública. v.21, n.3, p.200-10, 1987.

KOOGAN, L; LAROUSSE, P. Pequeno dicionário enciclopédico. Rio de Janeiro: Larousse, 1990.

KRUG, R. R., et al. Perfil dos profissionais de Educação Física que atuam em academias de musculação na região central da cidade de Criciúma/SC. Boletim Brasileiro de Educação Física, Brasília, v. 73, p.1- 9, 2009.

LEITE, P. F. Aptidão Física Esporte e Saúde. 3. ed. São Paulo: Editora Robe, 2000.

LIMA, M. A. A gestão da experiência de envelhecer em um programa para terceira idade: a UNATI/UERJ. In: VERAS, R. P. et al. Velhice numa perspectiva de futuro saudável. Rio de Janeiro: UERJ, UNATI, 2001.

LINS, R. G.; CORBUCCI, P. R.. A importância da motivação na prática de atividade física para idosos. Revista Estação Científica Online, n. 4, 2007.

LORET, A.: The needs and motivations of sport demanding.In: MEYER, M. V. Sport, leisure and physical education trends and developemnt. Aachen, p. 86-94, 1994.

MARQUES, A P.; CONDO, A.. A Fisioterapia na osteoartrose: uma revisão de literatura. Revista Brasileira de Reumatalologia. v. 38, n. 2, mar/abr 1998.

MATSUDO, S. M. Envelhecimento, atividade física e saúde. Revista Min. Educ. Fís., Viçosa, v. 10, n. 1, p. 195- 209, 2002.

MATSUDO, S. M. et al. Questionário internacional de atividade física (IPAQ): estudo de validade e reprodutibilidade no Brasil. Revista Brasileira de Atividade Física e Saúde, n.6, p.05-18, 2001.

MATSUDO, S. M.; MATSUDO, V. K. R. Prescrição e benefícios da atividade física na terceira idade. Revista Brasileira de Ciência e Movimento. v. 6, n. 9, p. 19- 30, 1992. 
MATSUDO, S.M.; MATSUDO, V.K.R.; BARROS NETO, T.L. Atividade física e envelhecimento: aspectos epidemiológicos. Revista Brasileira de Medicina do Esporte. v. 7, n.1, p.2-13, Jan/Fev, 2001

MAZO, G. Z. Universidade e terceira idade: percorrendo novos caminhos. Santa Maria: GZM, 1998.

MAZO, G. Z. Atividade Física, Qualidade de Vida e Envelhecimento. Porto Alegre: Sulina, 2008. 160 p.

MAZO, G. Z.; LOPES, M. A.; BENEDETTI, T. B. Atividade Física e o Idoso: Concepção Gerontológica. 3. ed. rev. e ampl. Porto Alegre: Sulina, 2009. 318 p.

MCAULEY, E. et al. Physical Activity, Self-Esteem, and Self-Efficacy Relationships In Older Adults: A Randomized Controlled Trial. Annals of Behavioral Medicine, v. 22, n.2, p.131-139, 2000.

MEURER, S. T. Motivos para a pratica de exercícios físicos, autoestima e a aptidão funcional de idosos: baseados na teoria da autodeterminação. Dissertação (Mestrado em Educação Física) - Centro de desportos. Florianópolis: Universidade Federal de Santa Catarina, 2010.

MIRANDA, C. J. M.; BATISTA, L. A. O prazer como determinante da aderência à atividade física por adolescentes, o caso do contexto escolar. In: CONGRESSO DE EDUCAÇÃO FÍSICA E CIÊNCIAS DO ESPORTE DOS PAÍSES DE LÍNGUA PORTUGUESA, 17, 1999, Florianópolis. Livro de Resumos, Florianópolis, 1999. p. 461.

NAHAS, M. V. Atividade Física, Saúde e Qualidade de Vida. 4. ed. Londrina: Midiograf, 2006.

NOBREGA, A. C. L. et al. Posicionamento oficial da Sociedade Brasileira de Medicina do Esporte e da Sociedade Brasileira de Geriatria e Gerontologia: Atividade Física e saúde no idoso. Revista Brasileira de Medicina do Esporte. v. 5, n. 6. 1999.

ODORIZZI, C. M. A. Uma pedagogia para a velhice: o desafio da construção de um trabalho com idosos no Brasil, 1996. Tese (Doutorado em Educação) - Pontifícia Universidade Católica, Porto Alegre, 1996.

OLIVEIRA, R. J. Exercício Maturidade Qualidade de Vida. 2. ed. Rio de Janeiro: Shape, 2002.

ORGANIZAÇÂO PAN-AMERICANA DE SAÚDE. Doenças crônico-degenerativas e obesidade: estratégia mundial sobre alimentação saudável, atividade física e saúde. Brasília: OPAS, 2002.

PATE, R. R. et al.Physical activity and public health - a recommendation from the Centers for Disease Control and Prevention and the American College of Sports Medicine. JAMA, n. 273, p. 402-7, 1995. 
POWERS, S. K.; HOWLEY, E. T. Fisiologia do exercício: Teoria e aplicação ao condicionamento e ao desempenho. 5. ed., São Paulo: Manole, 2006.

QUEIROZ, M. N. G.; SOUZA, L. K. Atividades de lazer em jovens e adultos: um estudo descritivo. Licere, Belo Horizonte, v. 12, n. 3, set./2009

RYDWIK, E.; FRANDIN, K.; AKNER, G. Effects of physical training on physical performance in institutionalised elderly patients (70+) with multiple diagnoses. Age Ageing. v. 33, n.1, p. 13-23, 2004.

SHEPHARD, R. J. The scientific basis of exercise prescribing for the very old. Journal of the American Geriatrics Society, USA, v. 38, n.1, p. 62-70, Jan. 1990.

SILVA, C. A.; LIMA, W. C. Efeito enéfico do Exercício Físico no controle metabólico do Diabetes Mellitus tipo 2 à curto prazo. Arq. Bras. Endocrinol. Metab. v.46, n..5, Oct. 2002

SPIRDUSO, W. Physical dimension of aging. Champaign, Illinois: Human Kinetics, 1995.

STELLA, F.et al. Depressão do idoso e atividade física. Revista Motriz. Rio Claro: v. 8, n. 3, p. 91- 98, Ago/Dez 2002.

TRIVIÑOS, A. N. S. Introdução à pesquisa em ciências sociais - pesquisa qualitativa em educação. São Paulo: Atlas, 1987.

VATTEN, L. J.; NILSEN, T. I.; HOLMEN, J. Combined effect of blood pressure and physical activity on cardiovascular mortality. J Hypertens, n.24, p.1939-1946, 2006.

WARBURTON, D. E. R.; NICOL, C. N.; BREDIN, S. S. D. Health benefits of physical activity, the evidence. Canadian medical association journal, v.174, n. 6, p. 801- 809, 2006.

WEINBERG, R. S.; GOULD, D. Fundamentos da Psicologia do Esporte e do Exercício. 2. ed. Porto Alegre: Artmed Editora, 2001. 560 p.

ZANATA, A. G; FONSECA, P. H. S. da. Prevalência de indivíduos com sintomas de dependência ao exercício no ambiente de academia de ginástica. Caderno de Educação Física: estudos e reflexões, Marechal Cândido Rondon, v. 7, n. 13, p. 33- 43, 2008.

ZAITUNE, M. P. A. et al. Fatores associados ao sedentarismo no lazer em idosos, Campinas, São Paulo, Brasil. Cadernos de Saúde Pública. v. 23, n. 6, 2007. 
Rodrigo de R. Krug, Moane Marchesan,

Julio Cesar R. da Conceição, Giovana Z. Mazo,

Gabriel A. Antunes e Jamile C. Romitti

\section{Endereço dos Autores:}

Giovana Zarpellon Mazo.

Centro de Ciências da Saúde e do Esporte

Universidade do Estado de Santa Catarina.

Rua Pascoal Simone 358 - Bairro: Coqueiros.

Florianópolis - SC - Cep: 88080- 350 